Submitted to ApJ

\title{
Circumbinary Gas Accretion onto a Central Binary: Infrared Molecular Hydrogen Emission from GG Tau A
}

\author{
Tracy L. Beck ${ }^{1}$, Jeffrey. S. Bary ${ }^{2}$, Anne Dutrey ${ }^{3}$, Vincent Piétu ${ }^{4}$, Steṕhane Guilloteau ${ }^{3}$, S. \\ H. Lubow ${ }^{1}$, M. Simon ${ }^{5}$, \\ tbeck@stsci.edu, jbary@colgate.edu, Anne.Dutrey@obs.u-bordeaux1.fr, \\ pietu@iram.fr, Stephane.Guilloteau@obs.u-bordeaux1.fr, lubow@stsci.edu, \\ michal.simon@sunysb.edu
}

\begin{abstract}
We present high spatial resolution maps of ro-vibrational molecular hydrogen emission from the environment of the GG Tau A binary component in the GG Tau quadruple system. The $\mathrm{H}_{2} v=1-0 \mathrm{~S}(1)$ emission is spatially resolved and encompasses the inner binary, with emission detected at locations that should be dynamically cleared on several hundred-year timescales. Extensions of $\mathrm{H}_{2}$ gas emission are seen to $\sim 100$ AU distances from the central stars. The $v=2-1 \mathrm{~S}(1)$ emission at $2.24 \mu \mathrm{m}$ is also detected at $\sim 30 \mathrm{AU}$ from the central stars, with a line ratio of $0.05 \pm 0.01$ with respect to the $v=1-0 \mathrm{~S}(1)$ emission. Assuming gas in LTE, this ratio corresponds to an emission environment at $\sim 1700 \mathrm{~K}$. We estimate that this temperature is too high for quiescent gas heated by X-ray or UV emission from the central stars. Surprisingly, we find that the brightest region of $\mathrm{H}_{2}$ emission arises from a spatial location that is exactly coincident with a recently revealed dust "streamer" which seems to be transferring material from the outer circumbinary ring around GG Tau A into the inner region. As a result, we identify a new excitation mechanism for ro-vibrational $\mathrm{H}_{2}$ stimulation
\end{abstract}

\footnotetext{
${ }^{1}$ The Space Telescope Science Institute, 3700 San Martin Dr. Baltimore, MD 21218

${ }^{2}$ Colgate University, Department of Physics \& Astronomy, 13 Oak Drive, Hamilton, NY 13346

${ }^{3}$ Université de Bordeaux, Observatoire Aquitain des Sciences de l'Univers (OASU), 2 rue de l'Observatoire, BP89, F-33271 Floirac Cedex, France

${ }^{4}$ IRAM, 300 Rue de la Piscine, 38406 Saint Martin d’Héres, France

${ }^{5}$ Stony Brook University, Department of Physics and Astronomy, Stony Brook, NY 11794
} 
in the environment of young stars. The $\mathrm{H}_{2}$ in the GG Tau A system appears to be stimulated by mass accretion infall as material in the circumbinary ring accretes onto the system to replenish the inner circumstellar disks. We postulate that $\mathrm{H}_{2}$ stimulated by accretion infall could be present in other systems, particularly binaries and "transition disk" systems which have dust cleared gaps in their circumstellar environments.

Subject headings: physical data and processes: accretion, accretion disks — stars: pre-main sequence — stars: winds, outflows, accretion — stars: formation planetary systems: protoplanetary disks — stars: individual (GG Tau A)

\section{Introduction}

Planet searches and corresponding imaging follow-up suggest that as many as $25 \%$ of extrasolar planets reside in binary or higher order multiple stellar systems (e.g. Patience et al. 2002; Eggenberger et al. 2004; Mugrauer et al. 2007; Takeda et al. 2008; Mugrauer \& Neuhäuser 2009). This multiplicity fraction is likely a lower limit, since most searches have preferentially selected the potential hosts to be single star systems. Such a high multiplicity fraction for exoplanet host systems indicates that despite the increased dynamical complexity of young multi-stellar systems, which lead to the truncation of individual circumstellar disks and the rapid clearing ( $\leq 1000 \mathrm{yr}$ ) of the inner regions of circumbinary(system) disks, reservoirs of material frequently survive long enough for planets to form. Stars commonly form in binary systems, so the fact that planets exist in these systems adds tremendously to the diversity and prevalence of planets in the Galaxy (Duquennov \& Mavor 1991; Ghez et al. 1993; Reipurth \& Zinnecker 1993; Leinert et al. 1993; Simon \& Prato 1995; Duchêne et al. 2007).

Gas flows through dust-cleared gaps in the disks of binary star systems can also provide important clues about the processes that could occur in the embedded planetary systems that open gaps. Planetary systems can be considered to be binaries of extreme mass ratios. Most of the mass gain of gas giant planets may occur by means of mass flow through the gap surrounding the orbit of the planet (Lubow et al. 1999; Lubow \& D'Angelo 2006; Ayliffe \& Bate 2010) The gas flow through gaps in young planetary systems and onto circumplanetary disks plays a crucial role in the formation of the young planets. The physics of this process can be better understood with direct observational constraints placed on the similar processes that occur in young binary star systems.

In order to understand the formation of planets in young binary and multiple systems, we first must understand the ways in which the stellar cores interact with the gas and 
dust in their circumstellar environment. Clues to the dynamics of these interactions and the structures in the circumstellar/system environment are revealed by spectral energy distribution measurements, high-spatial resolution observations, and hydrodynamic simulations (Jensen \& Mathieu 1997; Artymowicz \& Lubow 1994; Dutrey et al. 1994; Artymowicz \& Lubow 1996; Günther \& Kley 2002; Pascucci et al. 2008). For instance, simulations showing how young binary systems quickly clear gaps in the inner regions of circumbinary disks also describe how material will fall through these gaps in "high-velocity streams," eventually crashing into the individual circumstellar disks (Artymowicz \& Lubow 1996; Günther \& Kley 2002). High-spatial resolution millimeter and optical/near-infrared observations of nearby young binary systems like GG Tau A clearly show that optically-thin regions exist between the circumbinary disk and the stellar components (Dutrey et al. 1994; Guilloteau et al. 1999; Roddier et al. 1996; McCabe et al. 2002; Krist et al. 2002, 2005; Piétu et al. 2011). However, these observations also strongly hint at the presence of streamers of material falling through the optically-thin, dynamically unstable region (Piétu et al. 2011). More recently, a nearinfrared high-resolution image of scattered light from SR 24, a binary system in nearby the Ophiuchus star forming region, clearly indicates the presence of circumstellar disks orbiting both the primary and secondary and a spiral arm-like stream of material extending some 1600 AU from central sources toward a circumbinary disk (Mavama et al. 2010).

The replenishment of the tidally-truncated, inner circumstellar disks with material from the extended circumbinary(system) disks is likely to aid the formation of planets in many binary and multiple systems. Moreover, the recent discovery of Kepler-16b, an exoplanet that lies within the same orbital plane as its central binary host, demonstrates the potential for planets to form within circumbinary disks (Doyle et al. 2011). High-spatial resolution images capable of resolving emission from structures within a circumbinary region and near the inner circumstellar disks will be essential for studying the planet-building process in such systems. Constraining existing hydrodynamical models, which form our basis for understanding the evolution of young multiple systems and their host planets, would be a meaningful step in unraveling the complexity of star and planet formation in the binary environment.

As an exciting step toward this goal, we present the first spatially resolved image of molecular hydrogen gas located within the optically-thin, unstable region of the GG Tau A system. This image provides strong support for the existence of suspected accretion "streamers" in this system. The material falling onto the inner ambient gas appears to create a shock front located in the dynamically unstable circumstellar environment of the stars. We compare the ratios of multiple $\mathrm{H}_{2}$ lines to those predicted by models of shocked molecular hydrogen gas and conclude that the emission is consistent with shock heating. We use these data to estimate the mass and physical conditions of the emitting gas residing within the cleared region of the GG-Tau A's circumbinary disk. Finally, we discuss the phenomenon of shocked 
infalling gas in a binary system and the implications for similar systems such as transitional disks that potentially harbor planetary companions in their inner dust-cleared gaps.

\section{Observations}

Observations were obtained using the Near IR Integral Field Spectrograph (NIFS) at the Gemini North Frederick C. Gillette Telescope on Mauna Kea, Hawaii. NIFS is an image slicing IFU fed by Gemini's Near IR adaptive optics (AO) system, Altair, that is used to obtain 3-dimensional $\mathrm{x}, \mathrm{y}$, wavelength $(\lambda)$ spectral cubes at spatial resolutions of $\leq 0$ '. 1 with a 2 pixel spectral resolving power of $\mathrm{R} \sim 5300$ at $2.2 \mu \mathrm{m}$ (McGregor et al. 2003). The NIFS spatial field is $3^{\prime \prime} \times 3^{\prime \prime}$ in size and the individual IFU pixels provide 0 "' $1 \times 0 .{ }^{\prime \prime} 04$ spatial sampling. Data were obtained at the standard $K$-band wavelength setting for a spectral range of 2.010$2.455 \mu \mathrm{m}$. GG Tau A was bright enough to serve as its own wavefront reference stars for the facility adaptive optics system to guide at a $1000 \mathrm{~Hz}$ rate. All observations used for the final co-added datacube were acquired in natural seeing of better than $\sim 0$ ".85 for excellent AO correction.

The data for this project was acquired in queue observations during Fall 2009 under program ID GN-2009B-Q-40 through thin cirrus and good seeing. Observations of GG Tau A were executed over four different nights. The data reduction and analysis of the IFU dat-

acubes on GG Tau was identical to our past integral field spectroscopic studies (Beck et al. 2008, 2010). The individual reduced GG Tau A datacubes were re-sampled to $0^{\prime \prime} .04 \times 00^{\prime \prime} .04$ size pixels in the spatial dimensions. The exposures each had 40 second integration times, and the data was acquired with small dithers that were the same size as the width of the NIFS IFU slicing mirrors. The purpose of dithering the stars at integer slice mirror widths was to sample the stellar point spread function (PSF) in the cross dispersion direction in different pixels to improve the spatial sampling and mitigate the effect of the NIFS rectangular spatial elements. The final datacube on GG Tau A was constructed by shifting-and-adding 78 individual dithered exposures for a total on-source exposure time of 3210 seconds. To verify the $K$-band flux of the GG Tau A system and calibrate the imaging spectroscopy, supporting photometry was acquired in Sep. 2009 using the WIYN High-Resolution Infrared Camera (WHIRC; Meixner et al. 2010), using the $K_{s}$ filter and the United Kingdom Infrared Telescope (UKIRT) faint standard FS 18 for flux calibration. The derived $K_{s}$ magnitude for the combined GG Tau A brightness is $7.7 \pm 0.1$, which is consistent with all published photometry of the system.

By inspecting the wavelength calibration and sky emission line positions, we estimate that the absolute velocity accuracy of our individual IFU cubes for a given night of obser- 
vations is $\sim 9-12 \mathrm{~km} \mathrm{~s}^{-1}$ (roughly one third of a spectral pixel, or one sixth of a resolution element). For our observations, the accuracy of the NIFS IFU grating wheel repositioning was repeatable only to $\sim \pm 0.75$ pixels over the four nights of our observations. Hence, because of very weak signal from $\mathrm{H}_{2}$ emission in GG Tau A, data acquired over multiple nights was not subsampled and shifted and co-added in the wavelength dimension to preserve velocity resolution. As such, we estimate that our final emission line velocity centroid accuracy is affected by the grating repositioning and the straight co-addition of data that is slightly shifted in wavelength. As a result, the estimated absolute velocity accuracy of the GG Tau A data is at the level of 0.7 to 1.0 pixels, or $20-28 \mathrm{~km} \mathrm{~s}^{-1}$.

\section{Results}

Figure 1 presents the image of $2.12 \mu \mathrm{m} v=1-0 \mathrm{~S}(1) \mathrm{H}_{2}$ emission in the environment of GG Tau A. This image was constructed by summing six spectral pixels centered on the $\mathrm{H}_{2}$ emission at each spatial location in the field, after the continuum level was subtracted using a linear fit to the stellar continuum flux on each side of the emission feature. GG Tau Aa, the southern star, is centered in the image. Overplotted in blue are the $2 \mu \mathrm{m}$ continuum contours for both sources. The $\mathrm{H}_{2}$ emission is distributed around the binary, with obvious extensions of the emission to $\sim 70 \mathrm{AU}\left(0^{\prime \prime} .5\right)$ distances from GG Tau Aa. The strongest $\mathrm{H}_{2}$ emission arises from north-east of the system, about 0.2 away from the peak continuum position of GG Tau Ab. Given the projected separation of the GG Tau A binary, the $\mathrm{H}_{2}$ gas emission arises from dynamically unstable locations near the stars. In the image, the apparent decrease in $\mathrm{H}_{2}$ emission strength at the positions of the stars is likely not genuine, it results from decreased sensitivity to the faint $\mathrm{H}_{2}$ over the very bright stellar flux. If $\mathrm{H}_{2}$ at the level of the peak emission seen in Figure 1 existed at the location of GG Tau Aa, it would be less than $0.5 \%$ of the continuum flux and would be extremely difficult to detect after the continuum subtraction process.

A binned $2 \times 2$ image of the $\mathrm{H}_{2}$ emission from the environment of GG Tau A is shown in Figure 2a. In this image, the brightest $\mathrm{H}_{2}$ is saturated to emphasize the low level emission in regions surrounding the young binary. $\mathrm{H}_{2}$ "streamers" of emission are seen extending out to $\sim 0^{\prime \prime} 9$ distances from the central stars. Three positions are highlighted in the image Positions A, B and C. To the right in Figure 2b, the 1-D spatially integrated spectra (from 0 '.12 radius apertures) of the $2.12 \mu \mathrm{m} v=1-0 \mathrm{~S}(1)$ line are presented. The integrated signalto-noise ratios are $\sim 24,4$ and 16 for the $\mathrm{A}, \mathrm{B}$ and $\mathrm{C}$ spectra, respectively. The velocity of the

$\mathrm{H}_{2}$ emission in the A and B spectra are centered at $\sim-10 \mathrm{~km} \mathrm{~s}^{-1}$. Given the $\sim \pm 20-28 \mathrm{~km} \mathrm{~s}^{-1}$ absolute accuracy of our velocity calibration, this is not a statistically significant difference 
from the systemic radial velocity of GG Tau A of $18 \mathrm{~km} \mathrm{~s}^{-1}$ (Hartmann et al. 1986). These results are consistent with the previous high spectral resolution $(\mathrm{R} \sim 60,000)$ detection of $\mathrm{H}_{2}$ emission from the GG Tau A system (Bary et al. 2003). The spectrum extracted at the C position has a double-peaked profile shape, with a significant redshifted contribution to the line flux.

Figure 3 presents a spectrum of the $\mathrm{H}_{2}$ emission summed from spatial locations that have greater than $4.0 \times 10^{-17} \mathrm{~W} \mathrm{~m}^{-2}$ line flux in the $v=1-0 \mathrm{~S}(1)$ line. In addition to the $v=1-0 \mathrm{~S}(1)$ line at $2.12 \mu \mathrm{m}$, five other $\mathrm{H}_{2}$ emission transitions are detected, including the $v=2-1 \mathrm{~S}(1)$ line at $2.24 \mu \mathrm{m}$. The measured value of the $v=2-1 \mathrm{~S}(1) / v=1-0 \mathrm{~S}(1)$ line ratio is commonly used to determine the physical conditions of the emitting $\mathrm{H}_{2}$ gas. Assuming that the gas is in LTE, the Boltzmann relation and line ratio provide an estimate of the temperature of the emitting gas. For the spectra in spatial locations nearby to GG Tau A (Figure 3 ), we measure a value of $\sim 0.05 \pm 0.01$ for this line ratio and determine a corresponding gas temperature of $\sim 1700 \pm 100 \mathrm{~K}$. At this temperature and average $\sim 40$ AU distance from the central stars, it is unlikely that this gas is heated by blackbody emission from the stars. Given that the line ratio is also consistent with shock heating or high-energy photoexcitation in a dense medium $\left(n_{H} \geq 10^{4} \mathrm{~cm}^{-3}\right)$, both processes are potential mechanisms for stimulating the observed emission. However, given the spatial extent of the emission, the lack of detectable X-ray emission and relatively weak UV flux from the binary, we conclude that shock heating is the most likely excitation mechanism for the $\mathrm{H}_{2}$.

The detection of $v=2-1 \mathrm{~S}(1)$ emission at $\sim 0$.'2 distances from the central stars is a very high-contrast measurement. The peak flux in the brightest region of $v=1-0 \mathrm{~S}(1)$ emission is $0.6 \%$ of the continuum flux at the position of GG Tau Ab. The detected $v=2-1 \mathrm{~S}(1)$ emission is $5 \%$ of the flux of the 1-0 S(1) line. This discrete line emission is detected at $5 \sigma$ confidence, with a contrast ratio of $3 \times 10^{-4}$ with respect to the continuum flux level of GG Tau Ab. Our total emission line sensitivity is better than this because the stellar PSF of only the closer GG Tau Ab star in the binary is considered. We estimate the overall sensitivity at the $\sim 0$ '. 2 distance from both stellar PSFs to be $\approx 8 \times 10^{-5}$. At this sensitivity level, we are successfully probing the parameter space of the next generation planet survey instruments, such as those using extreme coronographic adaptive optics and non-redundant masking techniques (Beichman et al. 2010). This is very difficult, and is made possible primarily because we are searching for discrete, narrow emission lines where the adaptive optics halo structure can be accurately subtracted from the stellar point spread function over the small wavelength range. Our detection of $v=2-1 \mathrm{~S}(1)$ emission in the GG Tau A system highlights the success of using adaptive optics fed IFU spectroscopy to spatially resolve emission line morphologies within the inner $30 \mathrm{AU}$ circumstellar environments of bright young stars. 


\section{Discussion}

The GG Tau system was first spatially resolved into a hierarchical quadruple by Leinert et al. (1991). At the time of those observations, the stars in the GG Tau A component of the quadruple had a spatial separation of $0^{\prime \prime} .255$ at a position angle of $+9^{\circ}$. Our observations, acquired $\approx 20$ years later, show a system separation of $0.251 \pm 0.008$ at position angle $334 \pm 1$ (measured east of north). In the twenty years since its discovery as a binary, the GG Tau A stars have changed their orientation by nearly 35 degrees, just under one tenth of the total angular orbit (Köhler 2011). The fact that the separation has remained largely constant over a wide arc of the orbit means that the system is viewed close to pole on and either the orbit is nearly circular, or the components are near periastron where the apparent separation would vary little (Köhler 2011). The masses of the two stars in the GG Tau A system are estimated at $\sim 0.8$ and $\sim 0.7 M_{\odot}$ (total system mass of 1.28 \pm 0.08 ; White et al. 1999; Dutrey et al. 1994; Guilloteau et al. 1999). Hartmann et al. (1986) found the combined GG Tau A system to have a vsini of less than $10 \mathrm{~km} \mathrm{~s}^{-1}$, implying that the stellar components may also be viewed close to pole-on. Though, in order to explain the large 180 AU extent of the inner edge of the circumbinary ring, dynamical modeling of the system implies that the orbital eccentricity of GG Tau A may lie in the range of 0.2-0.4 and the stellar binary orbit may not be coplanar with the circumbinary ring (Beust \& Dutrey 2005; Köhler 2011). Yet, when the full GG Tau system is modeled as a quadruple then the need for non-coplanarity of GG Tau A with the circumbinary ring is removed (Beust \& Dutrey 2006).

The peak of the detected molecular hydrogen emission in the GG Tau A system is located at a position that is $\sim 0^{\prime \prime} \cdot 2-0$ '.25 away from both GG Tau Aa and Ab. Given the separation of the components of the binary of 0.25 , the tidally truncated inner circumstellar disks will not extend to this distance from the stellar positions. Hence, $\mathrm{H}_{2}$ detected at this position is too extended to be associated with either of the circumstellar disks that exist around the stars. Hydrodynamic models simulating the clearing of the inner regions around young star binaries clearly suggest that material at the observed location of $\mathrm{H}_{2}$ emission should be dynamically cleared on timescales of a few hundred years Artymowicz \& Lubow 1996; Günther \& Kley 2002). Though, the presence of material within the dynamically unstable region is not unexpected, given that GG Tau system possesses several indicators of accretion activity and the inner disks must be replenished to persist.

The circumbinary ring encircling GG Tau A was first detected in scattered light by Roddier et al. (1996). Since the time of the first scattered light images, higher sensitivity observations have revealed an inner distribution of dust within the circumbinary ring of

GG Tau A, extending to $\sim 0^{\prime \prime} .5$ north of the inner binary (Duchêne et al. 2004). This inner dust distribution corresponds in spatial location to the northern extension of $\mathrm{H}_{2}$ emission 
that we see. Our detected extended $\mathrm{H}_{2}$ emission can not merely be scattered line flux from a compact, centrally excited $\mathrm{H}_{2}$ emission environment because a central emission region would need to be hundreds to thousands of times stronger than our detected extended line emission region. To produce our measured extended $\mathrm{H}_{2}$ line flux, a central emission region would need to be at a level of more than a $30 \%$ of the continuum, and would be easily detectable over the central stellar flux. Moreover, if our detected $\mathrm{H}_{2}$ line emission arose from scattered line emission, we would also expect to detect $\mathrm{H}_{2}$ at the circumbinary ring, which is the location of the strongest and most easily detected scattered flux. We do not detect appreciable $\mathrm{H}_{2}$ emission from the circumbinary ring, to a detection limit of $8.0 \times 10^{-19} \mathrm{~W} / \mathrm{m}^{2} /$ pixel. The brightest region of $\mathrm{H}_{2}$ emission we detect seems to be spatially coincident with the inner region of dust-scattered light revealed in broad-band imaging, though the $\mathrm{H}_{2}$ emission must be excited locally within this environment rather than scattered.

Various high contrast observations of the GG Tau A system have reported the detection of "spokes" or "streamers" of material extending from the inner edge of the circumbinary ring inward toward the central binary system. These observations have been met with some level of skepticism because while ground-based studies seem to show some weak evidence for the streamers, images from the Hubble Space Telescope typically did not (Krist et al. 2005; McCabe et al. 2002). For the first time, Piétu et al. (2011) detected a possible streamer of dust emission in the mm wavelength map of the circumbinary ring around GG Tau A. This streamer of dust was detected at a $\sim 2 \sigma$ level of confidence, and it seems to extend from the northern edge of the circumbinary ring inward to the north side of the central binary. Figure 4 presents an image of our $\mathrm{H}_{2}$ gas emission around the GG Tau A system with the $1.1 \mathrm{~mm}$ dust continuum contours from Piétu et al. (2011) overplotted. Our $\mathrm{H}_{2}$ map also shows evidence of "streamers" of emission, and several of the $\mathrm{H}_{2}$ extensions are detected at high signal-to-noise. Interestingly, the location of the inner edge of the $\mathrm{mm}$ dust streamer corresponds precisely to the location where we detect the peak emission in molecular hydrogen gas. The presence of the peak $\mathrm{H}_{2}$ emission at a coincident location with the inner dust streamer lends further support that the streamer in the dust map is a real feature. Additionally, Guilloteau \& Dutrey (2000) report the existence of CO emission from these regions, which would also support the existence of streaming gas interior to the circumbinary dust ring.

In the environments of young stars, most excitation mechanisms considered for rovibrational $\mathrm{H}_{2}$ excitation involve stimulation by X-ray or UV flux from the central star, or shock excitation by material in an outflow. Bary et al. (2003) using the X-ray excitation models of Maloney et al. (1996), ignored the complexities of the binary system and assumed a centrally located X-ray/UV source incident on material located in a standard disk model (Glassgold et al. 2000) to predict a range of plausible $\mathrm{H}_{2}$ line fluxes for the GG Tau A system. 
Using $\log \mathrm{L}_{x}=29.4 \mathrm{ergs} \mathrm{s}^{-1}$, Bary et al. (2003) assumed that the X-ray energy was deposited in the upper atmosphere of a singular disk between 10 and 30 AU from the X-ray source. In the best case scenario, the X-rays were capable of stimulating roughly $70 \%$ of the observed $\mathrm{H}_{2}$ line flux in a high-resolution spectrum of GG Tau A. Bary et al. (2003) further assumed the UV flux from GG Tau to be similar to that of TW Hya and estimated the contribution of UV fluorescent $\mathrm{H}_{2}$ emission to be substantially less than the X-rays. However, they concluded that the combination of the limit of X-ray ionization and UV fluorescence might account for a significant portion of the $\mathrm{H}_{2}$ line flux in the GG Tau A system. With the spatial resolution and wavelength coverage of NIFS, we find that the gas is not distributed in the manner predicted by high-energy photon disk excitation models described by Bary et al. (2003); Bary et al. (2008). The $\mathrm{H}_{2}$ emitting gas extends farther from the sources than predicted and appears to display a morphology better described by gas participating in an accretion streamer than the central compact circumstellar disks illuminated by X-rays. In addition, the $v=2-1 \mathrm{~S}(1) / v=1-0 \mathrm{~S}(1)$ line ratio of $0.05 \pm 0.01$, measured at $\sim 40$ AU distances corresponds to $T \sim 1700 \pm 100 \mathrm{~K}$. This ratio can be accurately modeled by C-type shocks with reasonable pre-shock gas densities and shock velocities (see below; Le Bourlot et al. 2002). In light of these considerations, the lack of detectable X-ray flux from the GG Tau A system, and the spatial coincidence of the northern $\mathrm{H}_{2}$ gas with the possible dust stream (Figure 4), we conclude that this gas is most likely being shock excited by infalling material crashing into gas distributed throughout the circumstellar domain than stimulated by high-energy photons.

The measured emission line flux of the $v=1-0 \mathrm{~S}(1)$ transition and the estimated gas excitation temperature coupled with the partition function analysis from Herbst et al. (1996) allow us to determine the molecular hydrogen column density:

$$
N_{\text {tot }}=4.55 \times 10^{16} \cdot T \cdot\left(e^{6000 / T}-1\right) e^{956 / T} \cdot I_{\lambda} \cdot 10^{0.4 \mathrm{~A}_{\lambda}}
$$

where $I_{\lambda}$ is the measured line intensity (in $\operatorname{erg} \mathrm{cm}^{-2} \mathrm{~s}^{-1} \mathrm{sr}^{-1}$ ), $T$ is the gas temperature (assuming LTE), and $A_{\lambda}$ is the extinction to the emitting region, which we adopt to be 0.7 (White et al. 1999). The derived column density of $\mathrm{H}_{2}$ in the brightest knot of emission is $\mathrm{N}_{\text {tot }}=1.6 \times 10^{15} \mathrm{~cm}^{-2}$. The $\mathrm{H}_{2}$ emitting region sampled here was a square pixel with a $0^{\prime \prime} 1$ size. Hence these dimensions and the calculated $N_{\text {tot }}$ implies a mass of $\sim 10^{-12} \mathrm{M}_{\odot}$ in molecular hydrogen in this aperture, assuming a 140 pc distance to the Taurus molecular cloud. Assuming comparable column densities in regions of the $\mathrm{H}_{2}$ circumbinary material

\footnotetext{
${ }^{1}$ Bary et al. (2003) did not note that this value was taken as an upper limit since GG Tau A was not detected by ROSAT
} 
encompassing the system out to $\sim 0^{\prime \prime} 3$ distances, we estimate that the mass in $\mathrm{H}_{2}$ around GG Tau A is at least several $\times 10^{-11} \mathrm{M}_{\odot}$. Using the stellar parameters from GG Tau Aa and $\mathrm{Ab}$ from White et al. (1999) with the accretion rate relations from Muzerolle et al. (1998), we have used our measured $\operatorname{Br} \gamma$ line luminosity to derive mass accretion rates onto the stars. These accretion rates are $2.7 \times 10^{-8} \mathrm{M}_{\odot} \mathrm{yr}^{-1}$ and $2.4 \times 10^{-8} \mathrm{M}_{\odot} \mathrm{yr}^{-1}$ for GG Tau Aa and $\mathrm{Ab}$ respectively, consistent with past mass accretion rate estimates for the system (White et al. 1999). Not only does dynamical theory predict that the $\mathrm{H}_{2}$ should not be stable at our detected locations, but the mass accretion rate onto the stars is several orders of magnitude larger than the estimated mass of emitting $\mathrm{H}_{2}$ in the circumbinary environment. At this level of mass accretion, the extended $\mathrm{H}_{2}$ material must be continually replenished or it would be accreted rapidly onto the central disks and stars.

We have compared the detected fluxes of the $v=1-0 \mathrm{~S}(0), \mathrm{S}(1), \mathrm{S}(2)$ and $v=2-1 \mathrm{~S}(1) \mathrm{H}_{2}$ lines from the spectra of GG Tau (Figure 3) with the C-type shock models of Le Bourlot et al. (2002). We probed a range of pre-shock medium densities from $10^{3}$ to $10^{7} \mathrm{~cm}^{-3}$ and shock velocities from $10 \mathrm{~km} \mathrm{~s}^{-1}$ to $70 \mathrm{~km} \mathrm{~s}^{-1}$. The very slow shocks $\left(<10 \mathrm{~km}^{-1}\right)$ are ruled out with confidence, at these low velocities the shocks cannot produce the measured excitation temperatures greater than $1000 \mathrm{~K}$. Extremely fast high-density shocks also cannot reproduce the measured line fluxes, as these models result in greater excitation temperatures measured by the $v=2-1 \mathrm{~S}(1) / v=1-0 \mathrm{~S}(1)$ line ratio. We find that the optimal solutions in the models are for a pre-shock medium in the density range of $10^{3}-10^{5} \mathrm{~cm}^{-3}$ with a shock velocity of $20-50 \mathrm{~km} \mathrm{~s}^{-1}$. In GG Tau A, the accretion infall shock velocity would result from a combination of the gas free-fall velocity onto the system $\left(\sim 10 \mathrm{~km} \mathrm{~s}^{-1}\right)$ with a component from the Keplerian motion of material in the inner system. With these constraints, only the model calculations of shock velocities in the lower $20-30 \mathrm{~km} \mathrm{~s}^{-1}$ range would be consistent with the expected gas accretion kinematics for GG Tau A.

We speculate that $\mathrm{H}_{2}$ can also be stimulated into emission by mass accretion infall in other systems, such as young star binaries and systems with "transitional disks" that have dust cleared disk gaps that are thought to trace the onset of planet formation. In these latter systems, gas and dust from the outer distribution of disk material could accrete onto the inner regions, and $\mathrm{H}_{2}$ might be stimulated into emission from the shock excited free-falling material, which could have velocities in the $20-40 \mathrm{~km} \mathrm{~s}^{-1}$ range. For example, the GM Aur transition disk system has a dust-cleared gap in the inner disk that extends out to a distance of 20-25 AU from the central star (Dutrey et al. 2008; Hughes et al. 2009). Accretion streams of gas that free-fall from the $\sim 20$ AU outer ring onto an optically thin inner disk in the $\leq 1 \mathrm{AU}$ region (Espaillat et al. 2010) could have a velocity at the $30-40 \mathrm{~km} \mathrm{~s}^{-1}$ level, which would be fast enough to shock-excite the $\mathrm{H}_{2}$ into emission. 
In addition to the peak of $\mathrm{H}_{2}$ emission coincident with the inner dust streamer, extensions of $\mathrm{H}_{2}$ emission exist out to $\sim 0^{\prime \prime} .7$ (100 AU) separations from the central stars in GG Tau A (Position B in Figure 2). The detected $\mathrm{H}_{2}$ gas itself seems to exhibit "streamers" of emission that radiate outward from the central system. More sensitive data at both mm and near-infrared wavelengths could clarify the correlation between the streamers and the dust and gas species present within the system. Further study of the spatially resolved material apparent in the GG Tau A ring system would lead to a more detailed understanding of the general physics of mass accretion onto a central stellar binary. While this knowledge is directly applicable to other young star binaries, it also can serve to constrain the Keplerian motion in a broader base of models such as those that describe mass accretion onto proto-planets within circumstellar disks, as well as binary black holes in galaxy mergers and binary stellar remnants.

We are grateful to the Gemini North Observatory queue observers who acquired the data for this project, and to our NOAO and Gemini contact scientists Knut Olsen and Richard McDermid, who helped prepare this program for execution. Data for this project was acquired under Gemini Observatory program ID GN-2009B-Q-40. This study is based on observations obtained at the Gemini Observatory, which is operated by the Association of Universities for Research in Astronomy, Inc., under a cooperative agreement with the NSF on behalf of the Gemini partnership: the National Science Foundation (United States), the Science and Technology Facilities Council (United Kingdom), the National Research Council (Canada), CONICYT (Chile), the Australian Research Council (Australia), Ministério da Ciência e Tecnologia (Brazil) and Ministerio de Ciencia, Tecnología e Innovación Productiva (Argentina). 


\section{REFERENCES}

Artymowicz, P., \& Lubow, S. H. 1994, ApJ, 421, 651

Artymowicz, P., \& Lubow, S. H. 1996, Astrophysical Journal Letters, 467, L77

Ayliffe, B. A., \& Bate, M. R. 2010, MNRAS, 408, 876

Bary, J. S., Weintraub, D. A., \& Kastner, J. H. 2003, ApJ, 586, 1136

Bary, J. S., Weintraub, D. A., Shukla, S. J., Leisenring, J. M., \& Kastner, J. H. 2008, ApJ, 678,1088

Beck, T. L., Bary, J. S., \& McGregor, P. J. 2010, ApJ, 722, 1360

Beck, T. L., McGregor, P. J., Takami, M., \& Pyo, T.-S. 2008, ApJ, 676, 472

Beichman, C. A., Krist, J., Trauger, J. T., Greene, T., Oppenheimer, B., Sivaramakrishnan, A., Doyon, R., Boccaletti, A., Barman, T. S., \& Rieke, M. 2010, PASP, 122, 162

Beust, H., \& Dutrey, A. 2005, A\&A, 439, 585

-. 2006, A\&A, 446, 137

Doyle, L. R., Carter, J. A., Fabrycky, D. C., et al. 2011, Science, 333, 1602

Duchêne, G., Bontemps, S., Bouvier, J., André, P., Djupvik, A. A., \& Ghez, A. M. 2007, A\&A, 476, 229

Duchêne, G., McCabe, C., Ghez, A. M., \& Macintosh, B. A. 2004, ApJ, 606, 969

Duquennoy, A., \& Mayor, M. 1991, A\&A, 248, 485

Dutrey, A., Guilloteau, S., Piétu, V., et al. 2008, A\&A, 490, L15

Dutrey, A., Guilloteau, S., \& Simon, M. 1994, A\&A, 286, 149

Eggenberger, A., Udry, S., \& Mayor, M. 2004, A\&A, 417, 353

Espaillat, C., D’Alessio, P., Hernández, J., et al. 2010, ApJ, 717, 441

Ghez, A. M., Neugebauer, G., \& Matthews, K. 1993, AJ, 106, 2005

Glassgold, A. E., Feigelson, E. D., \& Montmerle, T. 2000, Protostars and Planets IV, 429 
Guilloteau, S. \& Dutrey, A. 2000, In "The formation of binary stars", eds. H. Zinnecker \& R.D. Mathieu, IAU Symposium, Vol. 200, 2001, by ASP, IAU Volumes (ISBN 1-58381-068-4).

Guilloteau, S., Dutrey, A., \& Simon, M. 1999, A\&A, 348, 570

Günther, R., \& Kley, W. 2002, A\&A, 387, 550

Hartmann, L., Hewett, R., Stahler, S., \& Mathieu, R. D. 1986, ApJ, 309, 275

Herbst, T. M., Beckwith, S. V. W., Glindemann, A., Tacconi-Garman, L. E., Kroker, H., \& Krabbe, A. 1996, AJ, 111, 2403

Hughes, A. M., Andrews, S. M., Espaillat, C., et al. 2009, ApJ, 698, 131

Jensen, E. L. N., \& Mathieu, R. D. 1997, AJ, 114, 301

Köhler, R. 2011, A\&A, 530, A126

Krist, J. E., Stapelfeldt, K. R., Golimowski, D. A., Ardila, D. R., Clampin, M., Martel, A. R., Ford, H. C., Illingworth, G. D., \& Hartig, G. F. 2005, AJ, 130, 2778

Krist, J. E., Stapelfeldt, K. R., \& Watson, A. M. 2002, ApJ, 570, 785

Le Bourlot, J., Pineau des Forêts, G., Flower, D. R., \& Cabrit, S. 2002, MNRAS, 332, 985

Leinert, C., Haas, M., Mundt, R., Richichi, A., \& Zinnecker, H. 1991, A\&A, 250, 407

Leinert, C., Zinnecker, H., Weitzel, N., Christou, J., Ridgway, S. T., Jameson, R., Haas, M., \& Lenzen, R. 1993, A\&A, 278, 129

Lubow, S. H., \& D’Angelo, G. 2006, ApJ, 641, 526

Lubow, S. H., Seibert, M., \& Artymowicz, P. 1999, ApJ, 526, 1001

Maloney, P. R., Hollenbach, D. J., \& Tielens, A. G. G. M. 1996, ApJ, 466, 561

Mayama, S., Tamura, M., Hanawa, T., Matsumoto, T., Ishii, M., Pyo, T., Suto, H., Naoi, T., Kudo, T., Hashimoto, J., Nishiyama, S., Kuzuhara, M., \& Hayashi, M. 2010, Science, 327,306

McCabe, C., Duchêne, G., \& Ghez, A. M. 2002, ApJ, 575, 974 
McGregor, P. J., Hart, J., Conroy, P. G., Pfitzner, M. L., Bloxham, G. J., Jones, D. J., Downing, M. D., Dawson, M., Young, P., Jarnyk, M., \& Van Harmelen, J. 2003, in Society of Photo-Optical Instrumentation Engineers (SPIE) Conference Series, Vol. 4841, Society of Photo-Optical Instrumentation Engineers (SPIE) Conference Series, ed. M. Iye \& A. F. M. Moorwood, 1581-1591

Meixner, M., Smee, S., Doering, R. L., Barkhouser, R. H., Miller, T., Orndorff, J., Knezek, P., Churchwell, E., Scharfstein, G., Percival, J. W., Mills, D., Corson, C., \& Joyce, R. R. 2010, PASP, 122, 451

Mugrauer, M., \& Neuhäuser, R. 2009, A\&A, 494, 373

Mugrauer, M., Neuhäuser, R., \& Mazeh, T. 2007, A\&A, 469, 755

Muzerolle, J., Hartmann, L., \& Calvet, N. 1998, AJ, 116, 2965

Pascucci, I., Apai, D., Hardegree-Ullman, E. E., Kim, J. S., Meyer, M. R., \& Bouwman, J. 2008, ApJ, 673, 477

Patience, J., White, R. J., Ghez, A. M., McCabe, C., McLean, I. S., Larkin, J. E., Prato, L., Kim, S. S., Lloyd, J. P., Liu, M. C., Graham, J. R., Macintosh, B. A., Gavel, D. T., Max, C. E., Bauman, B. J., Olivier, S. S., Wizinowich, P., \& Acton, D. S. 2002, ApJ, 581,654

Piétu, V., Gueth, F., Hily-Blant, P., Schuster, K.-F., \& Pety, J. 2011, A\&A, 528, A81+

Reipurth, B., \& Zinnecker, H. 1993, A\&A, 278, 81

Roddier, C., Roddier, F., Northcott, M. J., Graves, J. E., \& Jim, K. 1996, ApJ, 463, 326

Simon, M., \& Prato, L. 1995, ApJ, 450, 824

Takeda, G., Kita, R., \& Rasio, F. A. 2008, ApJ, 683, 1063

White, R. J., Ghez, A. M., Reid, I. N., \& Schultz, G. 1999, ApJ, 520, 811 


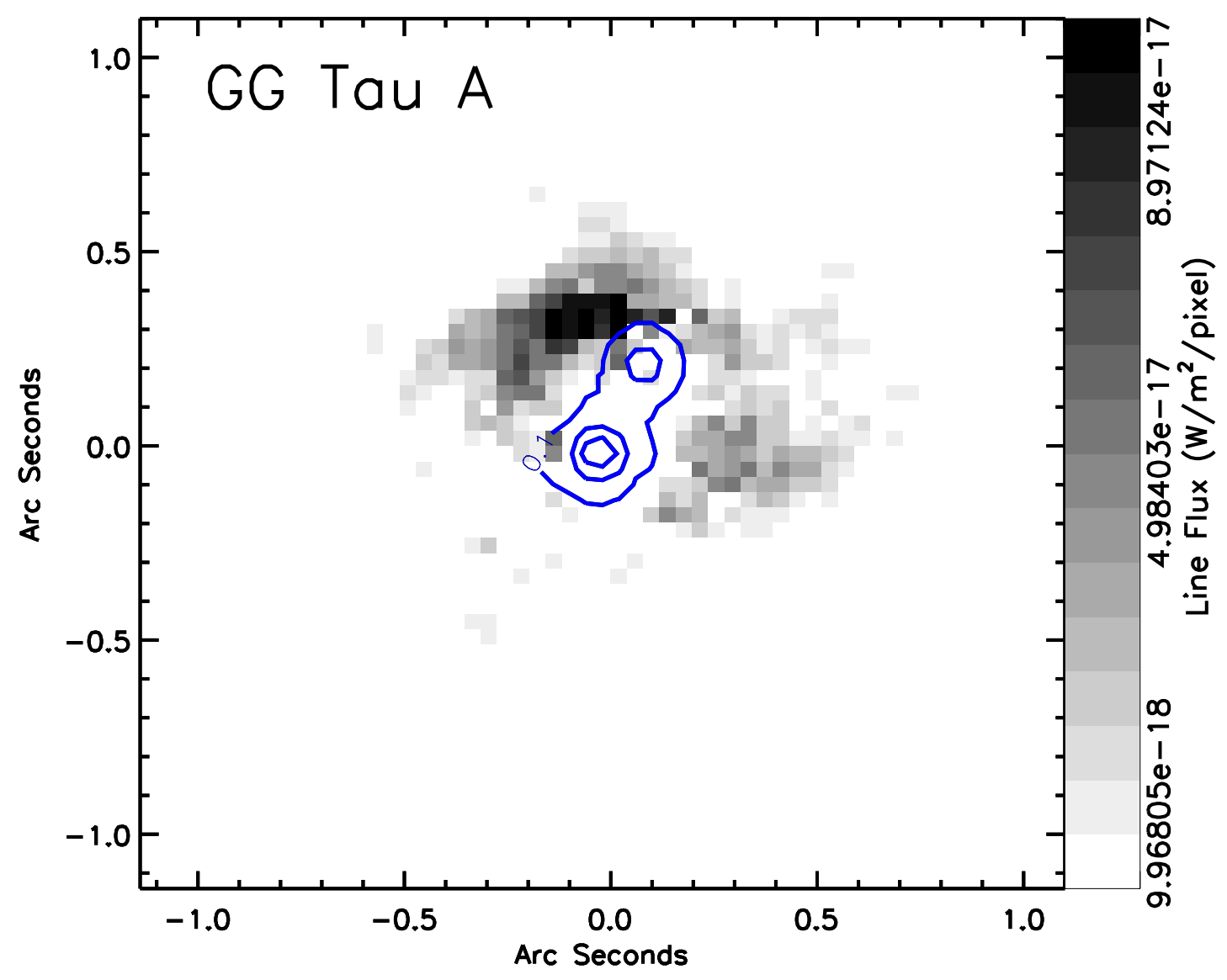

Fig. 1.- A Continuum subtracted grey scale image of $\mathrm{H}_{2}$ emission in the environment of GG Tau A binary, with continuum contours at $0.1,0.4$ and 0.7 times the peak continuum flux of GG Tau Aa overplotted in blue. The 0.7 and 0.4 contour levels were selected to highlight the continuum flux positions of the GG Tau Aa and Ab stars in this young binary. The $\mathrm{H}_{2}$ emission is distributed around the binary, with extensions to the emission out to $100 \mathrm{AU}$ (0." 7 ) distances. The apparent decrease in $\mathrm{H}_{2}$ emission at the positions of the stars is likely not genuine, it results from uncertainty in the continuum subtraction process to detect the faint $\mathrm{H}_{2}$ over the bright stellar flux. 


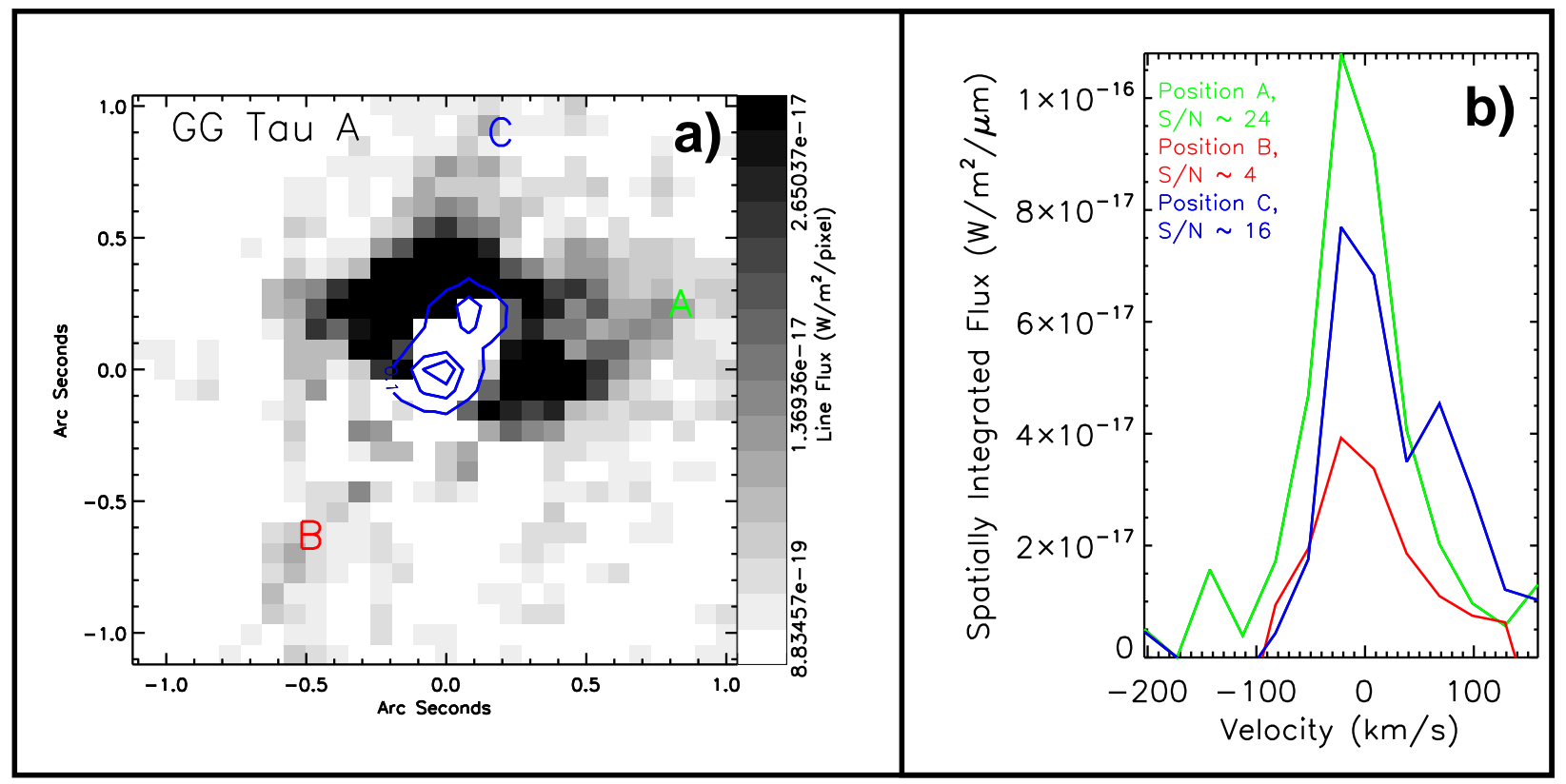

Fig. 2.- To emphasize the detection of low level line emission, a binned 2x2 image of the $\mathrm{H}_{2}$ emission from the environment of GG Tau A is presented in a). In this image the display is saturated to show the emission surrounding the young star binary. Three positions - Position $\mathrm{A}, \mathrm{B}$ and $\mathrm{C}$ - are highlighted in color in the image. To the right in b), the 1-D spatially integrated spectra of the $2.12 \mu \mathrm{m} v=1-0 \mathrm{~S}(1)$ line at the $\mathrm{A}, \mathrm{B}$ and $\mathrm{C}$ positions are presented. The $\mathrm{H}_{2}$ line emission is detected at significant signal-to-noise at spatial positions of more than 100AU from the central stars. 


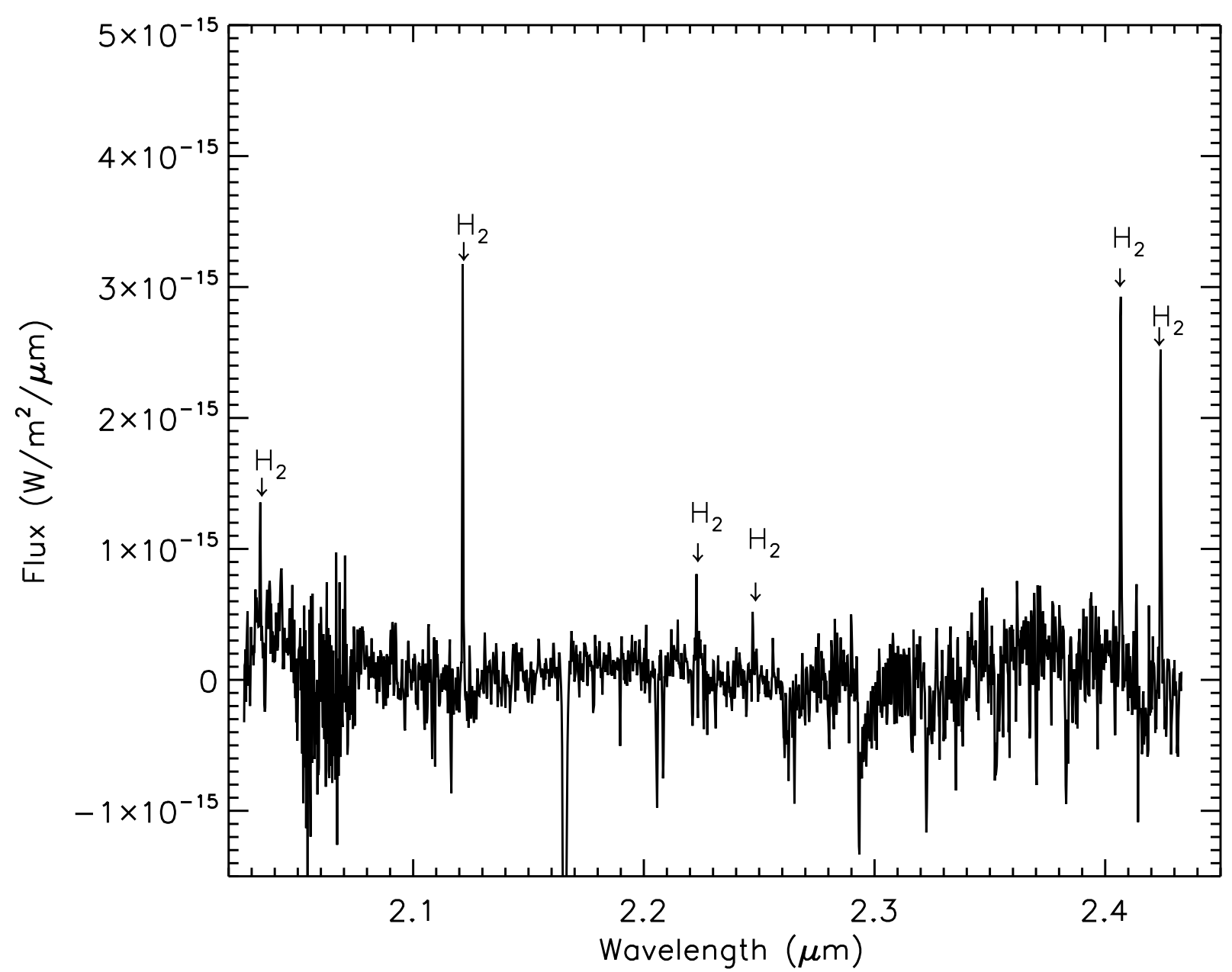

Fig. 3.- A spectrum of the $\mathrm{H}_{2}$ emission summed from regions of flux that are brighter than $4.0 \times 10^{-17} \mathrm{~W} \mathrm{~m}^{-2}$ (as seen in Figure 1). In addition to the $v=1-0 \mathrm{~S}(1)$ line at $2.12 \mu \mathrm{m}$, five other $\mathrm{H}_{2}$ emission transitions are detected, including the $v=2-1 \mathrm{~S}(1)$ line at $2.24 \mu \mathrm{m}$. The $v=2-1 \mathrm{~S}(1) / v=1-0 \mathrm{~S}(1)$ ratio we detect from the gas around GG Tau A is $\sim 0.05 \pm 0.01$, implying the presence of warm, $\mathrm{T}_{e x} \sim 1700 \mathrm{~K}$ gas at extended $\sim 40 \mathrm{AU}$ distances from the system. Residual stellar photospheric absorption features of $\mathrm{Al}+\mathrm{Mg}(2.11 \mu \mathrm{m}), \mathrm{Na}$ $(2.206 / 2.208 \mu \mathrm{m}), \mathrm{Ca}(2.26 \mu \mathrm{m})$ and $\mathrm{CO}(2.29-2.38 \mu \mathrm{m})$ are seen in this spectrum. 


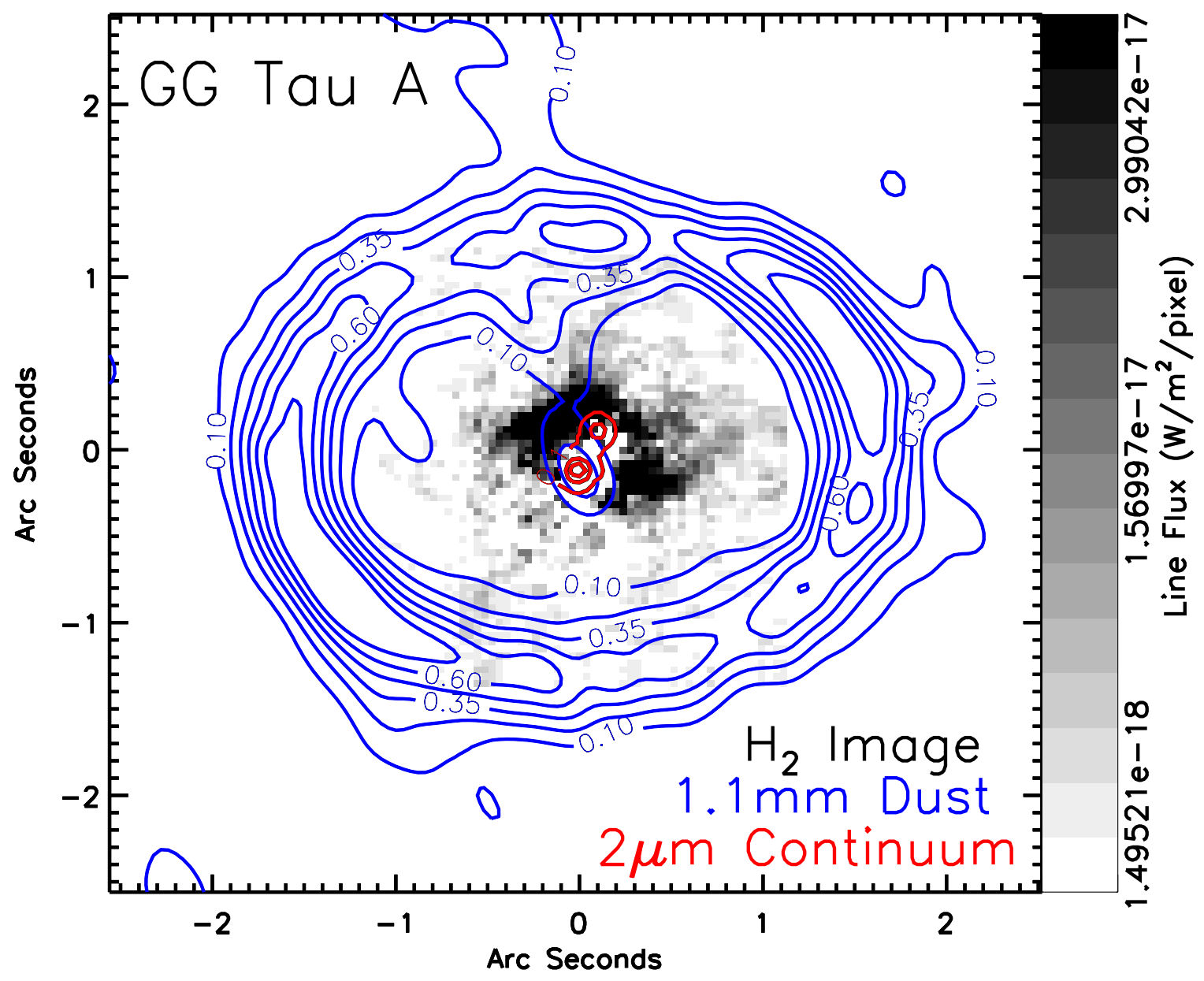

Fig. 4.- The continuum subtracted $\mathrm{H}_{2}$ image in the environment of GG Tau A (grey) with overplotted contours of the $1.1 \mathrm{~mm}$ dust continuum emission (blue) and the $2 \mu \mathrm{m}$ continuum flux (red). The brightest peak of molecular hydrogen emission corresponds to the spatial location of the dust "streamer" that is seen to extend within the circumbinary ring, which implies that both features are real and the $\mathrm{H}_{2}$ may be shock excited by mass accretion infall onto the binary stars. 